\title{
O USO DO SISTEMA DE INFORMAÇÃO NA ESTRATÉGIA SAÚDE DA FAMÍLIA: PERCEPÇÕES DOS ENFERMEIROS
}

\author{
Maria de Lourdes Custódio DUARTE ${ }^{\mathrm{a}}$, Janaina dos Reis TEDESCO ${ }^{\mathrm{b}}$, \\ Rodrigo Ritter PARCIANELLO
}

\section{RESUMO}

O estudo objetivou analisar a percepção dos enfermeiros sobre o uso do Sistema de Informação da Atenção Básica (SIAB), na Estratégia Saúde da Família (ESF), em cinco municípios do sul do país. Foram entrevistados cinco enfermeiros, e os dados foram analisados seguindo os passos da análise temática, fazendo emergir duas categorias: possibilidades de uso do SIAB e desafios enfrentados no cotidiano dos enfermeiros com o uso do SIAB. Salienta-se a importância das Coordenadorias Regionais de Saúde na organização de capacitações e discussões sobre o SIAB, além de assessorar os municípios na implantação efetiva desse sistema na atenção básica, envolvendo gestores, trabalhadores e comunidade. Um maior envolvimento desses atores na consolidação do SIAB facilitaria e qualificaria as ações de planejamento em saúde da população.

Descritores: Enfermagem familiar. Programa Saúde da Família. Sistemas de informação. Serviços de saúde.

\section{RESUMEN}

El objetivo del estudio fue analizar la percepción de las enfermeras sobre el uso del Sistema de Información de Atención Primaria (ISPC) en la Estrategia de Salud de la Familia (ESF) en cinco ciudades en el Sur. Cinco enfermeras fueron entrevistados y los datos fueron analizados siguiendo los pasos de análisis temático, surgen dos categorías: las posibilidades de utilizar el SIAB y desafíos que enfrenta en el diario de los enfermeros con el SIAB. Destaca la importancia de la Coordinación Regional de Salud en la formación de la organización y los debates sobre el SIAB, y ayudar a los municipios en la aplicación efectiva de este sistema en la atención primaria, la participación de gerentes, empleados y la comunidad. Una mayor participación de estos actores en la consolidación de facilitar la SIAB y calificar las acciones de la planificación de la salud de la población.

Descriptores: Enfermería de la familia. Programa de Salud Familiar. Sistemas de información. Servicios de salud. Título: Uso del sistema de información en la estrategia de salud de la familia: percepciones de las enfermeras.

\section{ABSTRACT}

The study aimed to analyze the nurses' perception about the use of the Information System of Primary Care (ISPC) in the Family Health Strategy (FHS) in five cities in the South. Five nurses were interviewed and data were analyzed following the steps of thematic analysis, two categories emerge: possibilities of using the ISPC and challenges faced in everyday nurse using the ISPC. Stresses the importance of the Regional Health Coordination in organizing training and discussions on the ISPC, and assist municipalities in the effective implementation of this system in primary care, involving managers, employees and community. Greater involvement of these actors in the consolidation of ISPC facilitate and qualify the actions of the population health planning.

Descriptors: Family nursing. Family Health Program. Information systems. Health services. Title: The use of information systems in family health strategies: nurses' perceptions.

\footnotetext{
a Professora Assistente da Universidade Federal do Pampa (Unipampa), Doutoranda pelo Programa de Pós-Graduação em Enfermagem (PPGENF) da Escola de Enfermagem da Universidade Federal do Rio Grande do Sul (UFRGS), Porto Alegre, Rio Grande do Sul, Brasil b Enfermeira. Especialista em Gestão do Trabalho pela Escola de Saúde Pública, Porto Alegre, Rio Grande do Sul, Brasil.

c Acadêmico de Medicina da Universidade Luterana do Brasil (ULBRA), Porto Alegre, Rio Grande do Sul, Brasil.
} 


\section{INTRODUÇÃO}

O Sistema de Informação da Atenção Básica (SIAB) foi criado em 1998 pelo Departamento da Atenção Básica/Secretaria da Atenção à Saúde, em conjunto com o Departamento de Informática e Informação do Sistema Único de Saúde (DATASUS). Essa ferramenta foi desenvolvida como um instrumento de apoio às equipes de saúde da família com o objetivo de identificar problemas individuais e coletivos do quadro sanitário de uma população, propiciando elementos para a análise da situação encontrada e subsidiando a busca de possíveis alternativas de encaminhamento ${ }^{(1)}$. Envolve tanto informações relativas ao processo saúde/doença, quanto às de caráter administrativo, todas essenciais para a tomada de decisão $0^{(2)}$.

Nesse contexto, inaugurou-se uma nova lógica de produção e de utilização da informação em saúde, tal como a utilização mais ágil e oportuna da informação e a produção de indicadores capazes de cobrir todo o ciclo de organização das ações de saúde a partir da identificação de problemas ${ }^{(3)}$. Assim, com a implantação do SIAB, os dados referentes às famílias passaram a ser registrados oficialmente, sendo possível estimar a cobertura média populacional da saúde da família, no Brasil ${ }^{(4,5)}$.

O SIAB, diferente de outros sistemas de informação em saúde, caracteriza-se por ser um sistema territorializado. Isso quer dizer que ele fornece indicadores populacionais (morbidade, mortalidade e de serviços) de uma determinada área de abrangência, facilitando a realização do diagnóstico de saúde, norteando o planejamento e avaliação de ações em saúde das equipes ${ }^{(6)}$.

No entanto, percebe-se a dificuldade que as equipes da Estratégia Saúde da Família (ESF) encontram para utilizar as informações produzidas e cadastradas no SIAB. Sabe-se que a alimentação do seu banco de dados, com informação das famílias cadastradas e o preenchimento dos relatórios, faz parte do trabalho diário dos profissionais desses serviços de saúde, e que o não preenchimento dessas fichas e dos relatórios prejudicam a avaliação de suas atividades, podendo levar ao descredenciamento da equipe e ao corte dos repasses/verbas junto ao Ministério da Saúde ${ }^{(7)}$. Dessa maneira, é atribuição de todos os componentes da equipe garantir a qualidade do registro das atividades nos sistemas nacionais de informação na Atenção Básica, contudo a realidade tem mostrado que são geralmente os enfermeiros os profissionais responsáveis pelo sistema local ${ }^{(2)}$.

O interesse em desenvolver o estudo e debater o tema surgiu no decorrer do trabalho em uma equipe da ESF, no qual se observou o desconhecimento sobre o SIAB e suas possibilidades de uso, notando-se manifestações de descaso com os dados coletados, bem como com o seu preenchimento no sistema. Além disso, notou-se que muitas vezes a alimentação dos dados no sistema ocorria de forma mecanizada, sem uma discussão prévia na reunião de equipe, tendo em vista que esses dados podem gerar informações importantes para o processo de trabalho nestes serviços de saúde.

Acredita-se que o não reconhecimento da importância do SIAB para subsidiar o processo de tomada de decisão faz com que esses processos não sejam devidamente reconhecidos por gestores e trabalhadores de saúde. É nesse contexto que o presente estudo justifica-se, pois se sabe que as informações em saúde são subsídios fundamentais para o reconhecimento da situação de saúde de um grupo social.

Nesse cenário, faz-se importante abordar as percepções dos enfermeiros sobre o uso do SIAB, provocando reflexões nos profissionais sobre a importância dessa ferramenta para a gestão das equipes da ESF. Assim, entende-se a importância do papel do profissional de enfermagem para o uso do SIAB, tendo em vista a sua condição de coordenador da equipe de enfermagem e muitas vezes, de maneira formal ou mesmo informal, de gestor das equipes da ESF.

Então, questiona-se: Qual a percepção dos enfermeiros sobre o uso do SIAB na Estratégia da Saúde da Família? A partir disso, o presente estudo teve como objetivo geral analisar as percepções dos enfermeiros da ESF sobre o SIAB, e, por objetivos específicos, identificar possibilidades e desafios no uso desse sistema pelos enfermeiros desses serviços.

\section{METODOLOGIA}

Estudo de abordagem qualitativa, de caráter exploratório-descritivo ${ }^{(8)}$, realizado em cinco municípios de pequeno porte de uma microrregião da Serra do Rio Grande do Sul (RS), que possuem características semelhantes, como a colonização alemã e o número de habitantes, em torno de cinco mil em cada município. 
Foi convidada a participar do estudo a totalidade de enfermeiros das Equipes de Saúde da Família dos cinco municípios, ou seja, seis enfermeiros, tendo em vista que apenas uma cidade possuía duas equipes da ESF cadastradas. O tipo da amostra foi intencional, por convite, tendo como critérios de inclusão o tempo mínimo de seis meses de vínculo com a ESF e estar trabalhando no período da coleta de dados.

O município que possuía dois enfermeiros teve um profissional excluído do estudo, pois o mesmo não atendia ao tempo de trabalho no serviço. Assim, foram entrevistados cinco enfermeiros que concordaram em participar do estudo.

A coleta dos dados ocorreu no mês de novembro de 2011, em horário acordado previamente, por meio de entrevistas semiestruturadas e individuais com cada enfermeiro, consistindo de três principais questionamentos: Qual é a sua percepção sobre o SIAB? Como se dá o uso do SIAB no seu processo de trabalho? Quais os desafios e possibilidades no uso cotidiano do SIAB?

As entrevistas foram gravadas e posteriormente transcritas na íntegra e analisadas. Procedeu-se à leitura integral das entrevistas, adotando-se o referencial de análise temática para apreciação crítica do conteúdo, buscando-se encontrar os trechos significativos para a constituição dos temas abordados em artigos consultados para pré-determinação dos eixos temáticos, em relação ao objetivo do estudo(s). Seguindo-se os passos de pré-análise e exploração do material, foi possível realizar a organização e a leitura repetida do corpus de pesquisa. Posteriormente, procedeu-se ao tratamento e interpretação dos resultados obtidos, descritos em unidades de registro e de contexto, que permitiram o agrupamento de ideias relevantes em categorias, representadas por dois eixos temáticos de discussão, a saber: possibilidades de uso do SIAB e desafios enfrentados no cotidiano dos enfermeiros com o uso do SIAB. Os sujeitos que participaram do estudo foram numerados de 1 a 5 na ordem em que foram entrevistados, e denominados pela letra $\mathrm{E}$.

A pesquisa foi aprovada pelo Comitê de Ética da Universidade Federal do Pampa (Unipampa), conforme Protocolo $n^{\circ}$ 047/2011, sendo considerados os aspectos éticos envolvendo seres humanos, em conformidade com o previsto na Resolução 196/96 do Conselho Nacional de Saúde ${ }^{(9)}$. Os participantes assinaram o Termo de Consentimento
Livre e Esclarecido após terem sido informados sobre os objetivos da pesquisa.

\section{APRESENTAÇÃO E ANÁLISE DOS RESULTADOS}

\section{Possibilidades de uso do SIAB}

Nesta categoria apresentam-se os dados coletados que remetem às possibilidades de uso do SIAB na perspectiva dos enfermeiros. Assim, para os entrevistados o SIAB viabiliza inúmeras possibilidades de uso no cotidiano de trabalho, como: 1) instrumento para coleta de dados; 2 ) instrumento de controle; 3 ) instrumento de informações; 4) instrumento que auxilia no diagnóstico local.

Os entrevistados apontaram o SIAB como um sistema de extrema importância e que facilita a coleta de dados da população pelos profissionais da $\mathrm{ESF}$

É um documento importantíssimo para a coleta de todos os dados das famílias. Ele deve ser bem preenchido e os dados bem coletados (E1).

Evidencia-se que o entrevistado entende a importância do SIAB para o seu cotidiano de trabalho na ESF, vislumbrando-o como um instrumento necessário para coleta de informações de saúde da comunidade atendida. Além da coleta das informações de saúde, é preciso que o profissional faça o preenchimento das fichas do SIAB de forma adequada, do contrário, a qualidade das informações estará bastante comprometida ${ }^{(10)}$.

O SIAB também serve como um instrumento de controle de saúde da comunidade da área de abrangência das equipes da ESF.

O SIAB é um controle de saúde sobre os dados da comunidade para as equipes que funciona muito bem, quando atualizado (E2).

O enfermeiro ressalta a importância do SIAB estar atualizado, caso contrário, as informações não servirão para que os profissionais as utilizem em favor da própria comunidade que atendem. Pois, somente com as informações atualizadas, os profissionais conseguirão saber quais problemas são realmente prevalentes entre as famílias e, dessa forma, poderão realizar o planejamento das ações de saúde. 
Nesse sentido, é importante disponibilizar as informações dos sistemas de forma organizada e de fácil acesso aos profissionais. Os Sistemas de Informação de Saúde (SIS) tornam-se ferramentas de apoio às atividades, para a tomada de decisão e aquisição de conhecimento, resultando em profissionais mais capacitados, criativos, capazes de mudar a realidade e melhorar o atendimento dos serviços de saúde ${ }^{(11)}$.

Outra possibilidade de uso do SIAB, na percepção de um enfermeiro, é que o mesmo serve como instrumento de informações da atenção básica, e que após sua digitação são enviadas para outras instâncias, ou seja, para os níveis estadual e federal.

Eu vejo o SIAB com um instrumento de informações. Todo o mês a gente digita no sistema as informações, para que elas sejam passadas para o nível estadual e federal(E3).

E lá a gente encontra então as informações de quantas famílias há no município, quantas pessoas, né, quantas doenças, qual o tipo de doença que tem mais porcentagem (E2).

Entende-se a importância e a possibilidade do SIAB em produzir informações de saúde da localidade em que a equipe da ESF está alocada, no entanto é necessário que, além de realizarem a digitação dos dados, os próprios profissionais usufruam desses dados coletados para o planejamento das ações de saúde.

Em um estudo realizado em Ribeirão Preto, foi identificado que os profissionais das equipes de ESF compreendem a finalidade do SIAB de produzir informações, no entanto, sua utilização aparece limitada ao registro, não sendo usado como instrumento gerencial básico para o planejamento e avaliação das ações ${ }^{(5)}$.

Muitas vezes, a utilização dos dados do SIAB acaba apenas gerando relatórios mensais, quem nem sempre são aproveitados pela própria equipe da atenção básica $^{(4)}$. Este sistema por vezes encontra-se verticalizado e centralizado, onde as equipes agrupam as informações, digitam e enviam para o nível central (Ministério da Saúde), sem realizar qualquer análise dos dados ${ }^{(12,13)}$.

Os entrevistados trazem o SIAB como um instrumento que possibilita fazer um diagnóstico local, facilitando aos profissionais conhecer a comunidade na qual atuam, proporcionando a identificação das necessidades de saúde das famílias e qualificando a assistência prestada à população.

\begin{abstract}
Através do SIAB temos a vantagem de saber sobre as gestantes, saber as patologias, sobre as idades de todo mundo. Ajuda no diagnóstico local mesmo, nos aproximando das famílias (E5).
\end{abstract}

A questão das vacinas também, né, a gente consegue ver quando começa a dar uma caída, nos faltosos (E3).

[...] a possibilidade de ter os dados ali de determinadas doenças, né, gestantes, crianças abaixo do peso, nesse

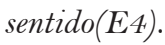

O conjunto das falas sugere que os dados coletados através do SIAB possibilitam o direcionamento do processo de trabalho das equipes da ESF, como é o caso dos faltosos para a vacinação e das crianças com baixo peso, demonstrando a importância do SIAB para a organização do serviço, através da identificação das necessidades da população $^{(5)}$. Assim, através da identificação das necessidades e das condições de saúde das famílias, existe a possibilidade de criação de vínculo entre profissionais e usuários de saúde. Esse encontro propicia um cuidado no domicílio, indo além das ações realizadas no serviço.

A partir das necessidades da comunidade visualizadas através do SIAB, os profissionais podem planejar suas ações, como é o caso das buscas ativas realizadas por meio de visitas domiciliares. Dessa maneira, o SIAB é um instrumento útil na identificação e avaliação das famílias, na construção de indicadores de saúde, na definição de prioridades, na organização do trabalho, na programação local e no direcionamento das visitas domiciliares ${ }^{(10,12)}$.

Para formular as políticas de saúde e para uma visualização global do sucesso dos projetos em execução, é preciso analisar e monitorar as condições de saúde da população, dispondo de um elenco de índices que avaliem diferentes dimensões do processo saúde/doença ${ }^{(14)}$.

Portanto, nessa categoria foi possível conhecer as possibilidades de uso do SIAB na percepção dos enfermeiros entrevistados de cinco municípios do Rio Grande do Sul. Para eles, o SIAB apresenta-se como um instrumento para coleta de dados, de controle e de informações, além de ser uma ferramenta que auxilia no diagnóstico local, ajudando na identificação das necessidades de saúde da comunidade. 


\section{Desafios enfrentados no cotidiano dos enfermeiros com o uso do SIAB}

Há inúmeras possibilidades de uso do SIAB no cotidiano de trabalho de enfermeiros que atuam na ESF, no entanto, algumas dificuldades são enfrentadas pelos enfermeiros relacionadas ao SIAB, tais como: 1) falta de capacitação para trabalhar com o SIAB; 2) falta apoio/assessoria para tirar dúvidas sobre o SIAB; 3) limitações do próprio sistema; e 4) falta de conhecimento dos outros profissionais sobre o SIAB.

A falta de capacitação para trabalhar com o SIAB foi verbalizada como a principal dificuldade no cotidiano de trabalho dos enfermeiros entrevistados, quando foram trabalhar na ESF.

Até agora não houve oportunidade de fazer capacitação sobre o SIAB. Utilizei as informações da graduação mesmo. No cotidiano é que vamos aprendendo (E1).

[...] sentei com uma das técnicas e ela começou a me ensinar, me mostrar como funcionava. Aprendi mexendo no programa mesmo (E2).

$\mathrm{O}$ conjunto de falas possibilita entender que não foi oferecido a esses entrevistados qualquer tipo de capacitação ou treinamento a respeito do SIAB. Esses profissionais buscam diferentes maneiras para entender o sistema, seja através de informações da própria graduação de enfermagem, seja através de ajuda dos colegas mais experientes da ESF.

Nesse sentido, uma pesquisa realizada em São Paulo em uma equipe da ESF identificou que os profissionais tinham inúmeras dificuldades em relação ao SIAB, sendo que a maioria dos entrevistados referiu a falta de capacitação, como principal limitador $^{(6)}$. Outras pesquisas também evidenciam a falta de treinamentos dos profissionais sobre o $\mathrm{SIAB}$ nas equipes da $\operatorname{ESF}^{(5,10,13,14)}$.

Os trabalhadores devem ser capacitados para conhecer o programa em sua totalidade: o preenchimento adequado das fichas, como buscar informações sobre a comunidade, confecção e análise de relatórios. A presença incipiente da educação permanente mostra a falta de uma política de qualificação dos profissionais nos municípios estudados, trazendo consequências para a qualidade das informações coletadas e analisadas.

É necessário que haja investimento tanto na educação permanente de toda a equipe, quanto nas discussões e interpretações dos dados coletados, pois a simples disponibilização de dados do SIAB para a equipe não é suficiente para sua utilização como informação na programação local ${ }^{(5)}$. Dessa maneira, com a ausência de treinamentos específicos para o preenchimento das fichas e uso dos impressos, a confiabilidade dos dados e a qualidade da informação podem ficar prejudicadas, o que pode significar um fator importante para a subutilização desse sistema por esses municípios ${ }^{(13,15)}$.

Associada à inexistência de capacitações, os entrevistados referem a falta de apoio/assessoria para sanar dúvidas em relação ao SIAB.

Tu não tens o apoio da coordenadoria. Cheguei a gente ligar pra lá e perguntar se a gente pode levar o nosso computador pra ver como instala e como trabalha (E3).

Falta assessoria, com quem dividir, ou planejar em cima dos dados do SIAB (E1).

Um entrevistado se dispôs a levar seu computador pessoal à coordenadoria, caso fosse necessário, para receber informações sobre o sistema. Já o outro sentiu dificuldade, pois não tinha com quem dividir as dúvidas, e muito menos fazer planejamento tendo como base os dados do SIAB. Assim, fica evidente o pedido de ajuda desses profissionais para as Coordenadorias Regionais de Saúde (CRS), que deveriam assessorar os municípios na implantação do SIAB.

Sabe-se que os municípios menores frequentemente apresentam dificuldades em relação à capacidade de gestão, mas também podem ter dificuldades adicionais para alocar recursos materiais e humanos para o trabalho com o SIAB. Nessas localidades pequenas, os responsáveis pelo sistema são majoritariamente funcionários estatutários e desempenhavam as mais variadas tarefas, de modo que o apoio da CRS para o aperfeiçoamento desses recursos humanos contribuiria para otimizar a utilização dos dados do $\operatorname{SIAB}^{(15,16)}$.

As limitações do SIAB relacionadas ao próprio programa ou ainda a informações que poderiam ser incluídas no sistema foram elencadas como dificuldades verbalizadas pelos enfermeiros entrevistados.

Acho que o SIAB é muito objetivo, podia ter um pouquinho mais de coisas(E5).

O SIAB é DOS, e DOS é muito difícil de trabalhar. Ele está defasado(E3). 
Os profissionais evidenciam falhas encontradas no software do SIAB como, por exemplo, o pequeno número de doenças codificáveis, que podem não colaborar para o real panorama da população $\operatorname{adscrita}^{(12)}$. Assim, entende-se a necessidade de readequar a ferramenta atual de coleta do SIAB, para que sua utilidade seja ampliada, vindo a agilizar as tomadas de decisão referentes aos processos de promoção e educação em saúde ${ }^{(17)}$.

Estudo realizado em 2009 recomenda algumas mudanças nas fichas, o que melhoraria a qualidade da coleta de dados ${ }^{(17)}$. Adicionado a isso, o SIAB é um sistema de nível nacional, o que limita enfocar as peculiaridades regionais, prejudicando a alimentação de dados importantes para a programação local ${ }^{(10)}$.

Os enfermeiros e os agentes comunitários de saúde são os profissionais que geralmente mostram maior envolvimento com o SIAB. Os outros profissionais permanecem mantendo um contato maior apenas com o preenchimento de suas fichas ${ }^{(5)}$ e outros nem sabem que o SIAB existe, e tampouco para que serve, como observa-se na próxima fala do entrevistado.

Os médicos nem chegaram a ser apresentados para o SIAB. Então era eu que lançava as informações no SIAB, porque eu sabia que era importante, mas eles nem ficaram sabendo pra que servia(E1).

Verifica-se a falta de conhecimento por parte do profissional médico sobre o SIAB e sua importância para atenção básica. Nesse sentido, o uso do SIAB parece se diferir entre os profissionais da equipe ${ }^{(6)}$. Assim, os baixos percentuais dos profissionais capacitados para a utilização do SIAB, principalmente os médicos, podem ter elevado o número de equipes que pouco utilizam o programa para planejamento das ações. Associado a isso, fatores como a inserção de profissionais sem o perfil adequado para o trabalho com a atenção primária podem contribuir para a baixa confiabilidade das informações geradas pelo sistema ${ }^{(5)}$.

Um dos achados de um estudo realizado em 2010 evidenciou que os profissionais da odontologia e da medicina utilizam pouco o SIAB e, quando utilizam, o fazem apenas para fins de preenchimento da sua produção. No entanto, o profissional de enfermagem é o que mais o utiliza como instrumento de análise da situação sociossanitária e epidemiológica, com os fins de diagnóstico e planejamento das ações ${ }^{(5)}$.
O conjunto das falas possibilita identificar que alguns fatores dificultam o trabalho dos enfermeiros com o SIAB, como a falta de capacitação, falta apoio/ assessoria para tirar duvidas sobre essa ferramenta, limitações do próprio sistema e falta de conhecimento dos outros profissionais sobre o SIAB.

\section{CONSIDERAÇÕES FINAIS}

Este estudo objetivou analisar a percepção dos enfermeiros sobre o uso do SIAB na ESF, identificando suas possibilidades e desafios de uso. Evidenciou-se que os profissionais entrevistados entendiam as inúmeras possibilidades de uso do SIAB, bem como sua importância para subsidiar seu processo de trabalho, no entanto verbalizaram a necessidade de capacitações para o uso adequado do sistema. Expressaram que o conhecimento adquirido sobre o SIAB na graduação era insuficiente, mas é esse conhecimento que tem feito diferença na prática profissional, tendo em vista a falta de atualizações sobre o assunto.

Ao finalizar este estudo, destaca-se a importância do SIAB para subsidiar melhores e mais precisas decisões na área da saúde, onde os desafios são diversos. Contudo, para que os profissionais possam utilizar essas informações no cotidiano, eles necessitam conhecer este sistema de informação e saber utilizá-lo. Nesse sentido, a relevância de ter dado voz aos enfermeiros, tendo em vista que a literatura destaca-os como os mais envolvidos com o SIAB.

Salienta-se a importância das Coordenadorias Regionais de Saúde na organização de capacitações e discussões sobre o SIAB, além de assessorar os municípios na implantação efetiva desse sistema na atenção básica, envolvendo gestores, trabalhadores e comunidade. Um maior envolvimento desses atores na consolidação do SIAB facilitaria e qualificaria as ações de planejamento em saúde nas comunidades.

Acredita-se que o SIAB seja um facilitador dos processos de trabalho na ESF, porém, para ser usado na sua plenitude, mudanças em relação à formação dos trabalhadores e ao apoio oferecido pelo estado e demais esferas deverão acontecer no país.

\section{REFERÊNCIAS}

1 Ministério da Saúde (BR), Secretaria de Atenção à Saúde, Departamento de Atenção Básica. Sistema de Informação da Atenção Básica - SIAB: indicadores 2003. Brasília (DF); 2004. 
2 Siqueira EA. Sistema de Informação da Atenção Básica (SIAB): instrumento de diagnóstico e planejamento [monografia]. Barbacena: Núcleo de Estudos em Saúde Coletiva, Faculdade de Medicina, Universidade Federal de Minas Gerais, 2010.

3 Shimizu HE, Reis LS. As representações sociais dos trabalhadores sobre o Programa Saúde da Família. Ciênc Saúde Col. 2011;16(8):3461-8.

4 Tillvitz LR, Souza RKT, Carvalho BG, Kasai MLHI, Nunes EFPA, Cordoni Junior L. Uso do sistema de informações na vigilância dos óbitos pelas Equipes de Saúde da Família em Londrina, PR. Cad Saúde Col. 2010;18(3):355-60.

5 Figueiredo LA, Pinto IC, Marciliano CSM, Souza MF, Guedes ABG. Análise da utilização do SIAB por quatro equipes da Estratégia Saúde da Família do município de Ribeirão Preto, SP. Cad Saúde Col. 2010;18(3):418-23.

6 Marcolino JS, Scochi MJ. Informações em saúde: o uso do SIAB pelos profissionais das Equipes de Saúde da Família. Rev Gaúcha Enferm. 2010;31(2):314-20.

7 Ministério da Saúde(BR). Portaria n. 648, de 28 de março de 2006: aprova a Política Nacional de Atenção Básica, estabelecendo a revisão de diretrizes e normas para a organização da Atenção Básica para o Programa Saúde da Família (PSF) e o Programa Agentes Comunitários de Saúde(PACS) [Internet]. Brasília (DF); 2006 [citado 2011 ago 25]. Disponível em: http://dtr2001.saude.gov. $\mathrm{br} / \mathrm{sas} / \mathrm{PORTARIAS} /$ Port2006/GM/GM-648.htm.

8 Minayo MCS. O desafio do conhecimento: pesquisa qualitativa em saúde. 10 ed. São Paulo: Hucitec; 2007.

9 Ministério da Saúde (BR), Conselho Nacional de Saúde. Resolução 196, de 10 de outubro de 1996: diretrizes e normas regulamentadoras de pesquisas envolvendo seres humanos. Brasília (DF); 1996.
10 Freitas FP, Pinto IC. Percepção da equipe de saúde da família sobre a utilização do Sistema de Informação da Atenção Básica (SIAB). Rev Latino-Am Enferm. 2005; $13(4): 547-54$.

11 Benito GAV, Licheski AP. Sistemas de Informação apoiando a gestão do trabalho em saúde. Rev Bras Enferm. 2009;62(3):447-50.

12 Silva AS, Laprega MR. Avaliação crítica do Sistema de Informação da Atenção Básica (SIAB) e de sua implantação na região de Ribeirão Preto, São Paulo, Brasil. Cad Saúde Pública. 2005;21(6):182 1-8.

13 Radigonda B, Conchon MF, Carvalho WO, Nunes FPA. Sistema de informação da atenção básica e sua utilização pela equipe de saúde da família: uma revisão integrativa. Espaço Saúde. 2010;12(1):3847.

14 Maia LDG, Corrêa JPR, Lopes ACFMM, Rodrigues Neto JF. Utilização do Sistema de Informação da Atenção Básica (SIAB) para o planejamento das ações pelas equipes da Estratégia de Saúde da Família do município de Montes Claros (MG). Rev Baiana Saúde Pública. 2010;34(2):359-70.

15 Barbosa DCM, Forster AC. Sistemas de Informação em Saúde: a perspectiva e a avaliação dos profissionais envolvidos na Atenção Primária à Saúde de Ribeirão Preto, São Paulo. Cad Saúde Col. 2010;18(3):424-33.

16 Colomé ICS, Lima MADS, Davis R. Visão de enfermeiras sobre as articulações das ações de saúde entre profissionais de equipes de saúde da família. Rev Esc Enferm USP. 2008;42(2):256-61.

17 Bittar TO, Meneghim MC, Mialhe FL, Pereira AC, Fornazari DH. O sistema de informação da atenção básica como ferramenta da gestão em saúde. RFO UPF. 2009;14(1):77-81.

\section{Endereço do autor / Dirección del autor / Author's address}

Maria de Lourdes Custódio Duarte

Rua Gonçalves Ledo, 20, ap. 203, Partenon

90610-250, Porto Alegre, RS

E-mail:malulcd@yahoo.com.br
Recebido em: 14.03.2012

Aprovado em: 13.09.2012 